$c$

Only a combined exposure of lunar silicates to the solar wind and micrometeorite impact can lead to the formation of lunar water, but not solar wind interaction alone g

\title{
ASTROCHEMISTRY
}

\section{Wet side of the moon}

Although the presence of water ice on the lunar surface has been acknowledged for several decades, its origin remains unclear. Several hypotheses have been put forward, including degassing from the primordial lunar interior, asteroid delivery and, as Ralf Kaiser, Jeffrey Gills-Davis and colleagues report in a new study, in situ alteration of surface minerals. Writing in the Proceedings of the National Academy of Sciences of the USA, the team from the University of Hawai'i presents experimental findings that support a mechanism for low-temperature in situ water release from protonated lunar silicates due to heating from micro-meteorite impacts.

Previous experiments designed to reveal the role of solar-wind protons in the formation of water on the moon have led to conflicting results. Water and hydroxyl groups were found after silicate samples were bombarded with protons under high-vacuum conditions, whereas no water was observed under ultraclean and ultrahigh vacuum conditions.

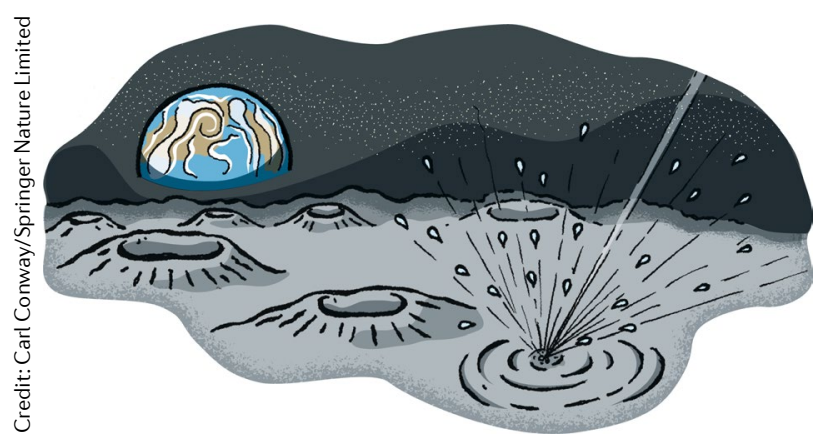

"This led to the hypothesis that the water molecules formed under 'dirty' high-vacuum conditions were actually contaminants condensing from the residual gas onto the cold silicate surfaces in the same way ice builds up in a refrigerator. Therefore, novel experiments under ultraclean conditions were necessary," explains Kaiser.

The group designed a new experiment in which anhydrous olivine $\left[(\mathrm{Mg}, \mathrm{Fe})_{2} \mathrm{SiO}_{4}\right]-\mathrm{a}$ mineral commonly used to simulate the lunar soil - is doped with hydrogen under ultraclean and ultrahigh vacuum conditions, over a temperature range of $10 \mathrm{~K}$ to $300 \mathrm{~K}$. In this case, deuterium ions were used to simulate the bombardment of lunar silicates by hydrogen ions from the solar wind. Deuterium, rather than hydrogen, was chosen to discriminate experimental signals arising from possible water-derived contamination. Upon warming of the protonated olivine samples, only $\mathrm{D}_{2}$ and no heavy water $\left(\mathrm{D}_{2} \mathrm{O}\right)$ was evolved, indicating that the silicates are capable of storing possible precursors to water at low temperatures, but that additional energy input is needed for the actual formation and release of water molecules.

In search of a higher-energy source capable of deriving water from the deuterated silicates, Cheng Zhu irradiated the protonated samples using a pulsed infrared laser, an energy source comparable to the heating developed by the impacts of micro-meteorites on the lunar surface. The researchers found that, at temperatures mirroring those on the lunar surface (10-300 K), heavy water was derived from the silicates. "Only a combined exposure of lunar silicates to the solar wind and micro-meteorite impact can lead to the formation of lunar water, but not solar wind interaction alone," states Kaiser. The irradiated samples were imaged by secondary electron imaging to identify the physical traces of water formation.

"Our work represents a particular analytical achievement, as the collaboration with physical chemists and planetary scientists helped the set-up of realistic experiments under ultraclean conditions," remarks Kaiser. "This methodology and proposed mechanism can lead to the formation of water not only on the moon, but on all airless bodies in our solar system and in extrasolar systems if silicates are present and the implanted solar wind particles can react upon micro-meteorite impact." These findings provide a fundamental explanation for how water is synthesized on the moon. Further experiments are needed to complete the story and reveal what other factors, including lithology and initial temperature of the lunar soil, as well as the impact of heavier ions, might play a role in the formation of water on the moon.

Tom Henshall

ORIGINAL ARTICLE Zhu, C. et al. Untangling the formation and liberation of water in the lunar regolith. Proc. Natl Acad. Sci. USA https:// doi.org/10.1073/pnas.1819600116 (2019) 